\title{
The AnyCorrectiveAction Stable Design Pattern
}

\author{
SHIVANSHU K. SINGH AND MOHAMED E. FAYAD, San Jose State University
}

\begin{abstract}
This paper aims at modeling a system that has to perform maintenance, by employing a Corrective Action and come up with a stable pattern, which can form a part of such a system, focusing at applying corrective action in a holistic way, rather than tying it on only one application specific context, which might cause potential impedance mismatch between process and workflows at a later stage. This ensures high reusability, ensuring that a design once created can be used to model any application, in any domain, thus making the task of designing more efficient, and the modeled system, largely domain independent. The goal of this paper is to design a Stable Pattern for Corrective Action and to model the system on the basis on an Enduring Business Theme (EBT). Here, the ultimate goal or the EBT is Maintenance and AnyCorrective action is what acts as a workhorse to achieve the goal of maintenance. In this paper, we have first designed a model, which defines the relationship of the Enduring Business Theme to Business Objects. We have then gone ahead with modeling an application scenario, which portrays the reusability of the developed stable pattern. A comparison of the Traditional Models and the Stable Model has also been included to describe how the latter overcomes to drawbacks of the former. Different models, such as, use cases, CRC cards, class diagrams and sequence diagrams have been used to give a better insight into the pattern and possible applications of it.
\end{abstract}

Categories and Subject Descriptors: D.2.7 [Software Engineering]: Distribution, Maintenance, and Enhancement - Corrections

General Terms: Design, Legal Aspects, Management, Verification

Additional Key Words and Phrases: Corrective Action, Design Pattern, Maintenance, Software Stability Model, Stable Design Pattern.

\section{INTRODUCTION}

The Traditional Model of software modeling does not take into consideration issues such as high reuse stability, scalability, impedance mismatch etc., and systems as well as patterns designed on those lines fail to be generic enough that they can be applied in any scenario regardless of the domain of application. Any software designed on traditional lines lacks the flexibility of being adapted to any other application. Any Software modeling performed by using that technique is never flexible and it will not be adaptable to changes. It's a static view of the system, and also lacks the ability to depict the dynamic nature of the system.

Also, in the software development industry, numerous projects have failed and they continue to do so. Adding to this, the ones which do not fail have huge costs of maintenance associated with them. This is primarily because there is a flaw in the analysis and design of those systems and the way they have been modeled. They have been modeled keeping one static configuration or only one application in mind and they lack the ability to dynamically adapt to changes. It is like constructing a big car manufacturing plant that produces just one car out of the facility and then re-structuring the whole plant again to produce the second car and so on, which clearly is an extremely inefficient way of doing anything.

So, now we are trying to model on the lines of making a system dynamic and instead of concentrating on a particular application as such, we are now concentrating upon a Stable Design Pattern of Corrective Action [1] and the Enduring Business Theme or EBT that it plans at implementing. The EBTs are realized through their workhorses, known as Business Objects or BOs, which are then applied to one or more

Author's address: Shivanshu K. Singh, Department of Computer Engineering, Charles W. Davidson College of Engineering, San Jose State University, San Jose, CA 95192, USA; email: shivanshukumar@gmail.com; Mohamed E. Fayad, Department of Computer Engineering, Charles W. Davidson College of Engineering, San Jose State University, San Jose, CA 95192, USA; email: mefayad@gmail.com

Permission to make digital or hard copies of all or part of this work for personal or classroom use is granted without fee provided that copies are not made or distributed for profit or commercial advantage and that copies bear this notice and the full citation on the first page. To copy otherwise, to republish, to post on servers or to redistribute to lists, requires prior specific permission. Preliminary versions of these papers were presented in a writers' workshop at the 17th Conference on Pattern Languages of Programs(PLoP). PLoP'10, October 16-18, Reno, Nevada, USA. Copyright 2010 is held by the author(s). ACM 978-1-4503-0107-7 
applications. We call this as the Stability Modeling [2] technique for software modeling. It gives us the ability to cater to various Business Themes and make the system domain independent rather than sticking to just one particular application of it [3]. We hereby attempt to show how the same system, created earlier by using traditional model can be modeled by using the stability model, thus giving it a larger purview and stability over time.

The stable design pattern AnyCorrectiveAction is based on the term Corrective Action, which generally refers to any counter measure taken by any party, who seeks Maintenance by the solution to any problem. Maintenance can be of different types: Preventive, Adaptive, Predictive and Corrective. Any Party decides upon taking any Corrective action, based on any evidence and the reason, and also the criteria that govern it. It involves one or more counter measures taken to prevent the recurrence of the problem or the reason for the corrective action. At the end of it, a log is produced, which serves as a basis for verification of the fact that the corrective action has been accomplished and it meets the ultimate goal of maintenance.

\section{PATTERN DOCUMENTATION}

2.1. Pattern Name: AnyCorrectiveAction Stable Design Pattern

The stable design pattern AnyCorrectiveAction is based on the term Corrective Action, which generally refers to any counter measure taken by any party, who seeks Maintenance by the solution to any problem. Maintenance can be of different types: Corrective, Adaptive, Predictive and Corrective. Any Party decides upon taking any Corrective Action, based on any evidence and the reason and also the criteria that govern it. It involves one or more counter measures taken to prevent the recurrence of the problem or the reason for the corrective action. At the end of it a log is produced, which serves as a basis for verification of the fact that the corrective action has been accomplished and it meets the ultimate goal of maintenance. AnyCorrectiveAction is a Business Object to a workhorse to realize the Enduring Business Theme of Maintenance.

\subsection{Known As}

Here we present a few patterns that might be confused with the pattern presented here. These are the patterns that must NOT be thought to be the same as the AnyCorrectiveAction pattern.

Betterment: The AnyCorrectiveAction pattern is sometimes also confused with the term betterment, i.e. making something that is there and making it better, but being confused as Corrective Action, but it's not quite the same, since betterment is about making something that is there, which is already in good shape and improving upon it. This is not what a corrective action does. Our pattern is employed, where we want to rectify a problem that exists, instead of making something which may be good, even better.

Prevention: Prevention is again, not about taking a corrective action. It is rather about taking preventive measures that help the system to avoid the occurrences of any new problems that would need a corrective action in the future. It is, hence, not the appropriate term for our pattern.

Correction: Correction, in some cases, can also mean the comments given for what should have been the right thing, instead what is currently there. [4] Here, we are not talking about it and since Correction encapsulates all such possibilities, i.e. the responses to any problem or way of doing things that currently exists, it is not accurate enough to be fit into the definition of the pattern in question. The term correction can also be the measure of changes made to anything to achieve the desired level/state, it might even be considered sometimes as a punishment for some wrong deeds. [5] Therefore, because the term 'correction' can have multiple meanings under different contexts, it is not accurate enough and it can't be used as the term which would represent the pattern AnyCorrectiveAction.

\subsection{Context}

The Stable Design Pattern, CorrectiveAction can be applied to any domain in which we have a board, which requires maintenance and which has a requirement of doing so, because of a problem that exists. For example, in military, there can be a corrective action taken, like initiating a court marshal on an officer, if the problem of smuggling of arms was the case and there was evidence that supported the act of smuggling. Generally applied in military domains, this pattern can now be applied across domains and by the means of Stability model, it has now attained a completely domain independent form. It can be applied 
to any organization i.e. a company that wants to raise working capital can make a corrective action of liquidating unused assets, which were bought earlier, but are not being used, or this can be applied to any university, where the university takes a corrective action of counseling students for not cheating, as a corrective action to maintain academic honesty or to counter the problem of plagiarism.

\subsection{Problem}

The problem at hand is to devise a design pattern that must incorporate the way corrective action is taken by any participant to overcome any issue or defect with the system that is recurring in nature i.e. a straightforward means of problem resolution does not yield a solutions and when there is an involvement of some kind of a binding authority with the problem resolution. There has to be a valid reason that prompts maintenance of the system for which the corrective action is required. From what has been observed in various disciplines and domains, a legally binding authority must approve the corrective action and has to fall under the periphery defined by the policies or Criteria set for by the authorities. Finally, Corrective Action must result into some that shows that the corrective action was taken and should also be supported by some form of documentation / or log. This is so that the process is recorded and the results can be captured and verified for the proper application of the pattern. The approving authority has to be displayed, the Evidence and the log of activities done, to prove that the corrective action was indeed taken.

Moreover another objective here is to capture the core knowledge related to one or more systems that would want to perform the corrective action process, in a way that a core knowledge is formed, that is enduring in nature and is not tied down to one specific domain or application context. It must be stable over time, i.e. must not become obsolete. The core knowledge thus captured should be such that it can help in spawning numerous applications on top of it realizes the true meaning of the pattern.

The various functional and non-functional requirements that need to be met in order to devise such a pattern are as follows:

\subsubsection{Functional Requirements}

Maintenance: Maintenance should correctly restore back the right state of the system. Maintenance would be achieved by some mechanism that is employed for this, but it's the job of maintenance as such, to make sure that the system now acts in the correct way that it should have after the corrective action was taken.

A Legal Entity/Participant: A legal entity or participant/governing authority needs to be involved in the process of carrying out corrective action. The entity specifies the criteria and constraints that govern the mechanism being used and the measures being taken in order to perform the corrective action. The one or more parties and persons must have someone with them, who has the expertise related to the domain in which the corrective action has to be done, so that the required corrective action(s) can be aptly identified, prioritized and instituted for action. Also, the party here is the authority, who establishes the fact that the corrective action was properly carried out by examining the logs and evidence generated. So, the expert(s) is (are) needed, so that it can be judged that whether the corrective action was properly applied or not.

Issuing Authority: There has to be some governing body that seeks maintenance to a problem and approves the corrective action. It cannot be done in an ad hoc manner by just anyone.

Criteria and constraints: AnyCorrectiveAction must be governed by the Criteria set forth by the issuing authority and must be performed under the boundary of one or more Criteria that have been set. It cannot go beyond the policies that drive the business; otherwise, the corrective action would result in other problems for which more corrective actions would be required.

Moreover, the criteria and constraints goes beyond just what a participant or the governing / issuing authority has defined with respect to themselves and their internal concerns; certain governing criteria can also come from the law of the land and the culture of the society that the system should cater to. Such criteria have to be met.

Criteria and the corrective action mechanism definition process: Defining is also one point of concern especially, when multiple authorities are involved in the formation of one or more Criteria. There has to be an effective coordination mechanism and a formal process of doing this, because the definition of criteria 
has to be carried out responsibly and effectively. Each participating member must review and approve the decisions being taken and for this, a formal process needs to be instituted.

Mechanism: Some Mechanism has to be used in order to perform CorrectiveAction and carry out Maintenance, so such a Mechanism must exist. A Mechanism would be governed by the Criteria defined by whoever seeks Maintenance. It is this Mechanism that achieves Maintenance. CorrectiveAction in this case can be a type of Mechanism. The reason for this kind of a requirement is let the system be flexible enough to accommodate any implementation decisions that need to be made when applying the pattern in any given context.

Type of Mechanism used: The type of mechanism used to attempt maintenance through CorrectiveAction has to be specific to the problem, which has to be addressed and it should be within context. Choosing the right mechanism is essential to the Corrective Action's appropriateness.

Proof: The corrective action must lead to an evidence or proof that shows that the corrective action was taken, otherwise, the purpose of corrective action is defeated and it cannot be made sure that whether the corrective action was taken in an appropriate manner or not.

Collection of Data: There should be provisions for capturing the related data, which results from the effects of the corrective action and must be captured through some documentation mechanism, maybe a Log, which can display the chronological order of the various actions and effects of this corrective action.

Reason: There has to be a well defined and identified reason for the corrective action to be performed and hence the maintenance to be undertaken. Generally for employing Corrective Action, the problem at hand is a recurring one. Thus, the reason has to be a recurring problem that occurs within the system. It has to be something internal to the system and external influences cannot be considered as reasons that require any corrective action on the system. One or more reason defines the type of Mechanism to be used to achieve Maintenance, depending upon what the reason is and the various factors and needs associated with them.

Type: Maintenance can be of different types: Corrective, Adaptive, Predictive and Corrective. Each one of these types will lead to special type of entities that needs to be included in the corrective action process. It is essential that such 'type' of Maintenance is clearly identified or a sub system is out in place that identifies the right kind of Maintenance that has to be done.

Entity: An Entity is something that is used by the Mechanism that is being employed, to perform Corrective Action and do Maintenance. So, an Entity being used by Mechanism has to be accessible and interface-able with the system, so that it can be used in performing the required tasks. Choosing the right entity is crucial here, since it may be governed by certain access regulations and there may be some other limitations to the use of it, so to maintain the cost effectiveness the entity must be chosen correctly. The Entity must therefore make the relevant meta data available upon request, so that the other parts of the system can properly decide, if it can be used or not and if so, can properly interface with it to get the job done.

\subsubsection{Non Functional Requirements}

Urgency: At any time, there may be a certain set of problems that need to be addressed and not just one such problem alone. In such a scenario, one problem may be more severe than the other and might require urgent attention. The system must also take into account such situations, where a bunch of corrective actions are required to be taken, but we need to prioritize them based on the urgency of the same. This may be decided upon by the issuing party as to which one to do first and which one the next.

Cost effectiveness: The corrective action being taken must be done in a cost effective manner. The system must not take forever to perform the tasks that are to be performed as a part of the process and still the entire process should be limited within a set amount of budget, so as to keep a balance between performance and costs incurred. Any extremes that occur here have the tendency of stalling the system, if it takes too long to finish the job or making it impossible for the management to keep the system in use, if it bears an enormous cost. 
Exactness: The corrective action taken should exactly conform to its description and must act fully and exactly as per the boundaries specified for it. Any more or less than the expected action, would render it useless and may even result in a situation, where one or more corrective actions are required to counter the ill-effects that it created in the processes of attempting the corrective action that was instituted. If the corrective action is not exact to its definition, more corrective actions would be required and that would result in increased cost, time and effort.

Reliability: The reliability of any corrective action is also desirable, because, if one is not sure about the outcome of the corrective action being taken, it cannot be predictable and hence would not be as effective in addressing the problem as it should be, thereby defeating the whole purpose of serving it only partially.

Also, the reliability and consistency of AnyLog and AnyEvidence is required, as if they are not reliable enough, there will not be a proof to the fact that the corrective action was taken and its effectiveness cannot be measured.

\subsection{Challenges and Constraints}

\subsubsection{Challenges}

1. Maintenance and Corrective Action as such, needs to be performed under certain constraints and by keeping in mind certain criteria. However, to come up with such criteria and to determine the right set of criteria is a challenge, while doing maintenance on the system.

2. There can be many criteria based on which, the Maintenance has to be performed, for any system. There may be some of them, which contradict each other; this is a challenge while defining the criteria.

3. Corrective Action can be applied in varied ways. Maintaining a system through Corrective Action would need certain mechanism to be used. Choosing the right mechanism that correctly enables performing the Corrective Action, is a challenge.

4. Accuracy is a big challenge, because there can be a corrective action for a problem, but things like learning and a learning plan for example, when used as corrective action, are heavily dependent upon learning abilities of the participants and so the corrective action though appropriate, but may not eventually result in a desired outcome and other measures might also be required. Hence, it is a challenge to make Any CorrectiveAction highly accurate.

5. There must be a proper reason for which maintenance is required and that too one that is of corrective in nature such that AnyCorrectiveAction needs to be employed. Sometimes, some thing close to the actual reason of a recurring problem can be confused to be the real cause of the problem that exists, because of which the system requires maintenance. In such a scenario, the efforts would be made to address this secondary reason, or the symptom, while the main reason would by mistake, get neglected and then even though the corrective action would be taken, it would fail to address the real reason, thereby rendering the whole exercise fruitless.

6. Recording the process of Maintenance and Corrective Action is a challenge in itself and the process, as well as the various factors considered in the process, findings etc should be recorded to make sure that the problem that caused the need for Maintenance is not repeated in the future.

7. The entities being used in the system for any purpose can pose a challenge for the system. If there are certain entities governed by access restrictions or usage policies, then to conform to these can be a big challenge. To add to it, when you consider many such entities being governed by different governing bodies/rules, it multiplies the complexity of the system. Hence, it has to be much more efficient in managing these entities and performing tasks like workload distribution etc., more the number of such requirements, the bigger the challenge would be.

8. Urgency of any corrective action may require the action to be taken within specific time and to meet stringent deadlines is a challenge in itself, on top of this, if there are more than one extremely high priority tasks to be done, then this efficiency has to be maintained at its best, else the system would fail to serve its purpose. Convoluting this with cost effectiveness raises even bigger challenges. 


\subsubsection{Constraints}

1. The Maintenance has to be performed within a certain set of criteria, depending upon the context in which the Maintenance is being performed.

2. Maintenance through Corrective Action can be performed only on a problem that is recurring and something which otherwise is not solvable.

3. A governing authority should approve Corrective Action before it is put into action. The Maintenance process that follows must document the procedure and log the results so that the execution of it can be verified.

4. Corrective Action can never go beyond the purview of the laws of the land and all the actions mandated as a part of that maintenance must conform to the law.

\subsection{Solution}

Before we dive into the solution of the problem at hand, we need to understand the various terms and concepts involved in coming up with the solution, and get to know the approach that we take

2.6.1. Software Stability Model (SSM) - Brief Overview The Software Stability Model is a way of designing and developing software and it does not go by the old school methods of software development. Instead of the application specific development approach, the software stability model focuses on the overall picture and it has a holistic approach to developing software, keeping in mind the ultimate goals of the software that we are developing, rather than concentrating on the specific application. The focus is on longevity of software and a generis development that can be reused in any related context, there by reducing the maintenance costs, which traditionally have accounted for more than $80 \%$ of the total cost of ownership of any enterprise class software.

Software Stability modeling is a modeling technique that uses the concepts of 'Enduring Business Themes' (EBT s), 'Business Objects' (BO s), and 'Industrial Objects' (IO s). These are classes that are usually differentiated into three different core layers, they are:

Enduring Business Themes (EBT s): EBTs come at the very core of the system and form the first layer. These represent the core knowledge of the system. They are also referred as the Stable Analysis Pattern, as they are the actual requirement of the system. EBTs remain stable over time i.e. they do not change, if the system has to be implemented in another application scenario. From a business point of view what this means is that through EBTs being the ultimate business goals, we want to keep the business goals constant, even if we change the way we offer the business in different ways over time.

Business Objects (BO s): BOs form the second layer of the system. These form the concrete classes of the system and are generally referred to as the "workhorses" of the system, under consideration. They are the enablers that realize the EBTs and contain the core design of the system/engine, and are stable over time too. They are also referred to as the stable design patterns.

The EBT(s) and BOs together form the stable core pattern that is not tied to a single domain or application context. This can now be used to build multiple applications on top of it. This application of the stable core in any given application scenario is done via the IOs.

Industrial Objects (IO s): The outermost layer of any software system developed on the lines of SSM, is made up of the IOs; this is the third layer. These represent those external applications that can be hooked to the pattern, which usually form the leaves of the system and which can be replaced without affecting the system. The BOs are customized and implemented through the IOs, there by enabling the system to be customized to any given application context and additional application specific functions added to the system, without having to change the core of it.

\subsubsection{Pattern Structure}




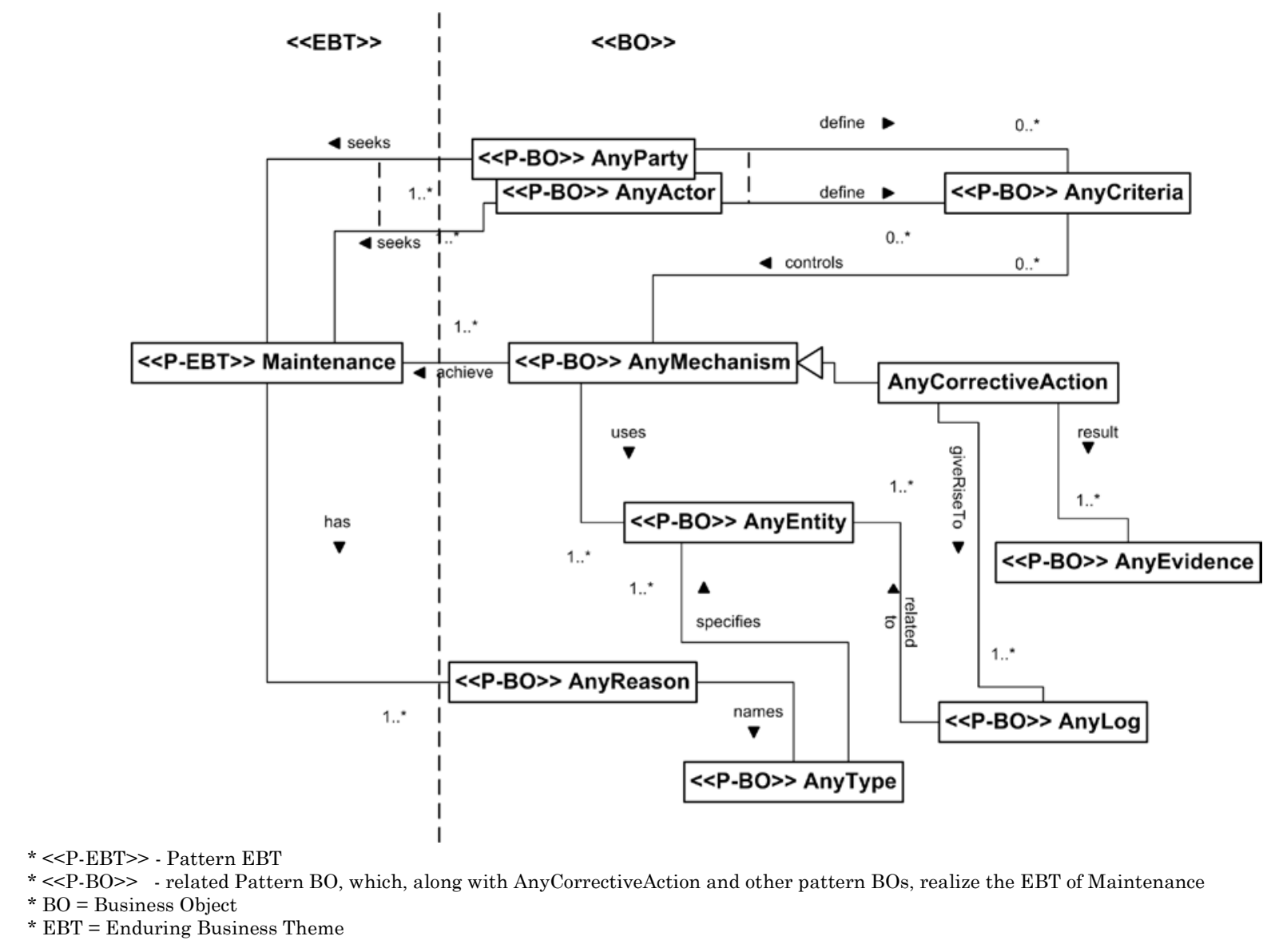

Fig. 1 - Pattern Class Diagram

\subsubsection{Class Diagram Description}

1. AnyParty or AnyActor seeks Maintenance, which is achieved through AnyMechanism

2. AnyParty or AnyActor defines AnyCriteria, which Controls AnyMechanism

3. Maintenance has AnyReason, which names AnyType, which specifies AnyEntity used by AnyMechanism

4. AnyCorrectiveAction results into AnyEvidence and gives rise to AnyLog

\subsubsection{Participants}

Classes:

AnyCorrectiveAction: any thought that is conceived by any actor.

Patterns:

Maintenance: represents the enduring concept of Maintenance, includes the operations and attributes of the same.

AnyEntity: $\quad$ refers to any objects that are being used in the system.

AnyActor: $\quad$ person, animal, software or hardware; something that acts on its own will.

AnyParty: $\quad$ any person, organization, country or political party.

AnyEvidence: something that forms the basis for AnyCorrectiveAction

AnyCriteria:

AnyType: refers to the basis that governs AnyMechanism through which Maintenance is achieved is the type of AnyMechanism and it specifies AnyEntity that shall be used by AnyMechanism 
AnyMechanism: the method employed to achieve Maintenance

AnyLog:

the record that is generated after AnyCorrectiveAction is taken, captures the various steps taken, the rationale and the results produced.

AnyReason: the problem that exists and which requires corrective action.

\subsection{CRC Cards}

The CRC cards are designed based on the guidelines as mentioned in [6, 7]. They help us in defining a complete picture of how a common set of operations, with individual responsibility of each class and the related operations served by it, can be utilized to form the basis of an application scenario. Such an application that is built on top of this pattern, utilizing one or more operations from the generic operations that are offered by the BOs, has been shown in subsequent section 5 (Applicability). The CRC-cards name the class, responsibility, and its collaborations. The CRC-cards also name a role for each class, which is useful for identifying the class responsibility. Each class should have only one and unique responsibility. The collaboration consists of two parts: clients and server. Clients are classes that collaborate and have relationship with the named class. Server contains all the services that are provided by the named class to its own clients. It is worth to point out that in documenting CRC-cards for stable patterns, we deal with any pattern that are included within the main pattern itself as a class. That is, each sub-pattern will be represented by a CRC-card that documents its responsibility and collaborations as a black box. To avoid any confusion, and for simplicity, we do not care about how the sub-pattern handle its reasonability according to its internal structure, all what we care about here is that this sub-pattern will perform the task as a black box, leaving the other details to the second abstraction level of the pattern description.

\begin{tabular}{|l|l|l|}
\hline \multicolumn{2}{|c|}{$<<$ P-EBT $>>$ Maintenance(Maintenance) } \\
\hline \multicolumn{1}{|c|}{ Responsibility } & \multicolumn{1}{|c|}{ Collaboration } \\
\hline \multirow{2}{*}{ To achieve consistency } & \multicolumn{1}{|c|}{ Clients } & \multicolumn{1}{c|}{ Server } \\
\cline { 2 - 3 } & $\begin{array}{l}\text { AnyParty } \\
\text { AnyActor }\end{array}$ & $\begin{array}{l}\text { maintain() } \\
\text { renew0 } \\
\text { increaseLifeSpan0) }\end{array}$ \\
\hline Attributes: & \\
\hline type, duration, degree, frequency, phase & \\
\hline
\end{tabular}

\begin{tabular}{|l|l|l|}
\hline \multicolumn{2}{|c|}{$<<$ P-BO>> AnyEntity (AnyEntity) } \\
\hline \multicolumn{1}{|c|}{ Responsibility } & \multicolumn{1}{|c|}{ Collaboration } \\
\hline \multirow{2}{*}{ to help in accomplishing a task } & \multicolumn{1}{|c|}{ Clients } & \multicolumn{1}{c|}{ Server } \\
\cline { 2 - 3 } & $\begin{array}{l}\text { AnyMechanism } \\
\text { AnyType }\end{array}$ & $\begin{array}{l}\text { describe() } \\
\text { modify0 } \\
\text { helpAccomplishTask0 }\end{array}$ \\
\hline Attributes: & \multicolumn{2}{|c|}{} \\
\hline name, domain, state, size, description &
\end{tabular}

\begin{tabular}{|l|l|l|}
\hline \multicolumn{2}{|c|}{$<<\mathrm{P}-\mathrm{BO}>>$ AnyCorrectiveAction(AnyCorrectiveAction) } \\
\hline \multicolumn{1}{|c|}{ Responsibility } & \multicolumn{1}{|c|}{ Collaboration } \\
\hline \multirow{2}{*}{$\begin{array}{l}\text { To take counter measure and produce } \\
\text { evidence of it }\end{array}$} & \multicolumn{1}{|c|}{ Clients } & \multicolumn{1}{c|}{ Server } \\
\cline { 2 - 3 } & $\begin{array}{l}\text { AnyMechanism } \\
\text { AnyEvidence } \\
\text { AnyLog }\end{array}$ & $\begin{array}{l}\text { takeCorrectiveMeasure( } \\
\text { restore() } \\
\text { produceLog( } \\
\text { resultEvidence( })\end{array}$ \\
\hline Attributes: & \multicolumn{2}{|l}{} \\
\hline duration, name, type, serialNumber, purpose & \multicolumn{2}{|c|}{} \\
\hline
\end{tabular}




\begin{tabular}{|c|c|c|}
\hline \multicolumn{3}{|c|}{$<<$ P-BO $>>$ AnyActor (AnyActor) } \\
\hline Responsibility & \multicolumn{2}{|c|}{ Collaboration } \\
\hline \multirow[b]{2}{*}{ to think and take action } & Clients & Server \\
\hline & $\begin{array}{l}\text { Maintenance } \\
\text { AnyCriteria }\end{array}$ & $\begin{array}{l}\text { takeAction() } \\
\text { defineCriteria() } \\
\text { seekMaintenance() }\end{array}$ \\
\hline \multicolumn{3}{|l|}{ Attributes: } \\
\hline \multicolumn{3}{|c|}{ name, address, occupation, idNumber, dateOfBirth } \\
\hline
\end{tabular}

\begin{tabular}{|l|l|l|}
\hline \multicolumn{2}{|c|}{$<<$ P-BO $>>$ AnyType (AnyType) } \\
\hline \multicolumn{1}{|c|}{ Responsibility } & \multicolumn{1}{|c|}{ Collaboration } \\
\hline \multirow{2}{*}{ specify the entity to be used } & \multicolumn{1}{|c|}{ Clients } & \multicolumn{1}{c|}{ Server } \\
\cline { 2 - 3 } & $\begin{array}{l}\text { AnyReason } \\
\text { AnyEntity }\end{array}$ & $\begin{array}{l}\text { specifyEntity() } \\
\text { signify( } \\
\text { determine( })\end{array}$ \\
\hline Attributes: & \multicolumn{2}{|c|}{} \\
\hline name, usage, classification, structure, value \\
\hline
\end{tabular}

\begin{tabular}{|l|l|l|}
\hline \multicolumn{2}{|c|}{$<<$ P-BO $>>$ AnyReason (AnyReason) } \\
\hline \multicolumn{1}{|c|}{ Responsibility } & \multicolumn{1}{|c|}{ Collaboration } \\
\hline $\begin{array}{l}\text { to serve as the cause for which } \\
\text { maintenance is required }\end{array}$ & $\begin{array}{l}|c| \\
\text { Maintenance } \\
\text { AnyType }\end{array}$ & $\begin{array}{l}\text { Clients } \\
\text { nameTyper } \\
\text { reflect) } \\
\text { corroborate0 }\end{array}$ \\
\hline Attributes: & \multicolumn{2}{|c}{} \\
\hline context, validity, significance, stimulus, name
\end{tabular}

\begin{tabular}{|l|l|l|}
\hline \multicolumn{2}{|c|}{$<<$ P-BO $>>$ AnyCriteria (AnyCriteria) } \\
\hline \multicolumn{1}{|c|}{ Responsibility } & \multicolumn{1}{|c|}{ Collaboration } \\
\hline \multirow{2}{*}{ to constrain any action being taken } & \multicolumn{1}{|c|}{ Clients } & \multicolumn{1}{c|}{ Server } \\
\cline { 2 - 3 } & $\begin{array}{l}\text { AnyParty } \\
\text { AnyActor } \\
\text { AnyMechnism }\end{array}$ & $\begin{array}{l}\text { control() } \\
\text { restrict() } \\
\text { formBasis0 }\end{array}$ \\
\hline Attributes: & \multicolumn{2}{|}{} \\
\hline domain, type, clauseNumber, name, effect & \\
\hline
\end{tabular}

\begin{tabular}{|l|l|l|}
\hline \multicolumn{2}{|c|}{$<<$ P-BO $>>$ AnyEvidence(AnyEvidence) } \\
\hline \multicolumn{1}{|c|}{ Responsibility } & \multicolumn{1}{|c|}{ Collaboration } \\
\hline \multirow{2}{*}{ serve as a proof } & \multicolumn{1}{|c|}{ Clients } & \multicolumn{1}{|c|}{ Server } \\
\cline { 2 - 3 } & AnyCorrectiveAction & $\begin{array}{l}\text { prove() } \\
\text { justify0 } \\
\text { assert) }\end{array}$ \\
\hline Attributes: & \\
\hline proof, timestamp, type, domain, assertion, reliability \\
\hline
\end{tabular}




\begin{tabular}{|l|l|l|}
\hline \multicolumn{2}{|c|}{$<<$ P-BO $>>$ AnyParty(AnyParty) } \\
\hline \multicolumn{1}{|c|}{ Responsibility } & \multicolumn{1}{|c|}{ Collaboration } \\
\hline \multirow{2}{*}{ To manage } & \multicolumn{1}{|c|}{ Clients } & \multicolumn{1}{c|}{ Server } \\
\cline { 2 - 3 } & $\begin{array}{l}\text { Maintenance } \\
\text { AnyCriteria }\end{array}$ & $\begin{array}{l}\text { takeAction() } \\
\text { defineCriteria() } \\
\text { seekMaintenance) }\end{array}$ \\
\hline Attributes: & \multicolumn{2}{|}{} \\
\hline partyName, purpose, motto, type, location, size
\end{tabular}

\begin{tabular}{|l|l|l|}
\hline \multicolumn{1}{|c|}{ Responsibility } & \multicolumn{1}{|c|}{ Collaboration } \\
\hline \multirow{2}{*}{ To list a chronological sequence } & \multicolumn{1}{|c|}{ Clients } & \multicolumn{1}{c|}{ Server } \\
\cline { 2 - 3 } & $\begin{array}{l}\text { AnyEntity } \\
\text { AnyCorrectiveAction }\end{array}$ & $\begin{array}{l}\text { document() } \\
\text { record0 } \\
\text { storeInfo) }\end{array}$ \\
\hline Attributes: & \multicolumn{2}{|}{} \\
\hline name, size, type, media, logNumber, timestamp \\
\hline
\end{tabular}

\begin{tabular}{|l|l|l|}
\hline \multicolumn{2}{|c|}{$<<$ P-BO $>>$ AnyMechanism (AnyMechanism) } \\
\hline \multirow{2}{*}{ Responsibility } & \multicolumn{1}{|c|}{ Collaboration } \\
\cline { 2 - 3 } to attempt doing maintenance & $\begin{array}{l}\text { Maintenance } \\
\text { AnyCriteria }\end{array}$ & $\begin{array}{l}\text { Clients } \\
\text { operate() } \\
\text { conduct) } \\
\text { achieve() }\end{array}$ \\
\hline Attributes: & \multicolumn{2}{|c|}{} \\
\hline reason, name, effectiveness, strength, type & \\
\hline
\end{tabular}

\subsection{Consequences}

The AnyCorrectiveAction Stable Design Pattern presents us with many challenges that have to be tackled. In order to meet those challenges and to fulfill them, we would need to design the system taking into consideration, certain capabilities that the system must have and doing so might result in many consequences that the system will face. Here are a few of those:

1. To be able to support a large amount of media for storing AnyLog generated by the system, it would need to have the capabilities for interfacing with the different types of media that are available. This would result in the system being of a large size, a considerable part of which would be something, which deals with this interfacing only, than anything else.

2. Also, if the user is to define the way to handle the storage, then we need to have the support for different storage mechanisms. If these storage facilities are on the Internet and need an access mechanism, then the system needs to cater to that too, and things like access rights management and other issues also need to be implemented.

3. These features may also call for support for plug-ins and an appropriate API would be needed, so that the consumer can make use of the available options within the system, as well as add his/her custom devices, by integrating the necessary plug-ins into the system.

4. Doing so, we may also have to consider many things like security, because we would allow the system to be customized by the client to a great extent and hence whatever the plug-in does need to be monitored 
or approved before it can be added, or alternatively such things have to be 'legally' taken care of and further investigation needs to be done in this area.

5. For the system to be cost effective and to deal with the issues like critical corrective actions, especially in those cases, where more than one corrective actions are of a very high priority and it is necessary to handle both of them as quickly as possible, then we need to empower the system with efficient scheduling capabilities, where the system can effectively decide which entity to use and at what time and for how long. The system needs to plan out for minimum access of the entities, if they are to be paid for on a pro-rata basis and hence the system should be able to arrange the set of activities, on the fly, in a way which strikes a balance between cost and performance, while staying within the cost and time constraints.

\subsection{Applicability}

This section describes one of the numerous application scenarios in which the AnyCorrectiveAction can be employed. The way we approach patterns is that we consider them as the stable core knowledge or core infrastructure, based on which a system can be designed. Thus the EBTs and the BOs together make up this stable core. Multiple applications can then be built on top of this stable core. In the case of AnyCorrectiveAction, for example, there can be several applications that can be built around the core concept of achieving Maintenance by the use of corrective action, it can be in military, academic institutions, business, hospitals and many more. This is done by the developed of highly specialized pieces of code called the IOs. The IOs customize the BOs for any pattern, to adapt them for a specific applications context. So while the EBT+BO core is generic and can be applied across multiple application contexts, the IOs are specific to a context and application. Described here is one such application that can be generated on top of the stable pattern.

\subsubsection{Application: Company trying to reduce losses}

This application is about a company, which is undergoing losses, because some of its employees lack minimum attendance. So, in order to reduce these losses incurred because of low attendance, the company takes corrective action in that regard. Over here, the company under question or 'Company' as referred to in the example, is suffering Losses due to poor attendance of employees in the offices. More and more people are slacking on work and eventually this amounts to poor quality of work and thus losses. Loss is the reason that the company has in order to seek corrective action. While they can take many steps, certain Policies or criteria dictate that a certain code of conduct has to be followed at all time. The company has a Policy that a Warning would be issued to any employee in case the attendance is found short. The Employee then undergoes the corrective action of counseling to motivate and guide him/her to be more devoted to his/her work. This corrective action (Counseling) leads to an improvement in the Attendance and the Attendance itself serves as the Evidence that supports that it got improved by the application of the corrective action. The balance sheet serves as the log that reports the losses and drawing a comparison, it can be observed that the losses decrease as the corrective action is applied. 


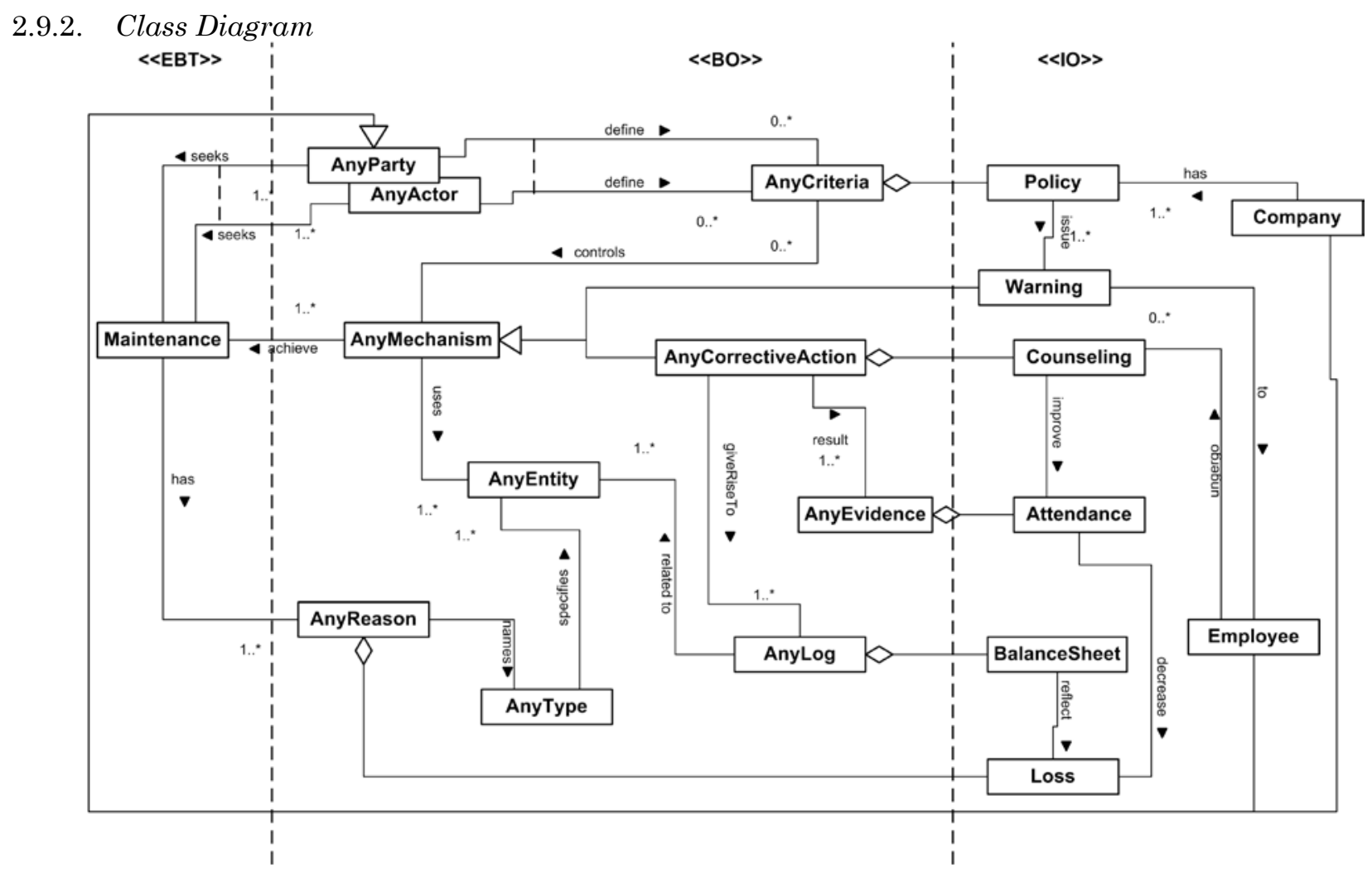

Figure 2 - Application 1 Class Diagram

\subsubsection{Class Diagram Description}

1. AnyParty(Company) or AnyActor seeks Maintenance, which is achieved through AnyMechanism(Warning)

2. AnyParty(Company) or AnyActor defines AnyCriteria(Policy), which Controls AnyMechanism(Warning)

3. Maintenance has AnyReason(Loss), which names AnyType, which specifies AnyEntity used by AnyMechanism(Warning, corrective action, counselling)

4. AnyCorrectiveAction(Counselling) results into AnyEvidence(Attendance increase) and gives rise to AnyLog(BalanceSheet - results are reflected in the balancesheet)

5. Company has a Policy, which issues one or more Warning to Employee

6. Employee undergoes Counseling, which imporves Attendance

7. Attendance decreases Loss

8. BalanceSheet reflects the Loss (and thus the increase / decrease)

2.9.4. Use Case

\begin{tabular}{|c|c|c|c|}
\hline Title & \multicolumn{3}{|c|}{ Reduce Losses Due To Improper Attendance } \\
\hline $\mathrm{Id}$ & \multicolumn{3}{|l|}{1.1} \\
\hline Actor & \multicolumn{3}{|l|}{ Roles } \\
\hline AnyParty & \multicolumn{3}{|c|}{ Company, Employee } \\
\hline Class & Type & Attribute & Operation/Interface \\
\hline Maintenance & EBT & $\begin{array}{l}\text { duration, } \\
\text { degree }\end{array}$ & renew() \\
\hline
\end{tabular}




\begin{tabular}{|c|c|c|c|}
\hline AnyReason & BO & $\begin{array}{l}\text { validity, } \\
\text { significance, } \\
\text { stimulus }\end{array}$ & corroborate() \\
\hline AnyParty & $\mathrm{BO}$ & $\begin{array}{l}\text { partyName, } \\
\text { motto, } \\
\text { type, } \\
\text { size }\end{array}$ & defineCriteria() \\
\hline AnyMechanism & BO & $\begin{array}{l}\text { name, } \\
\text { effectiveness, } \\
\text { strength }\end{array}$ & achieve() \\
\hline AnyEntity & BO & $\begin{array}{l}\text { description, } \\
\text { name }\end{array}$ & helpAccomplishTask 0 \\
\hline AnyCriteria & $\mathrm{BO}$ & $\begin{array}{l}\text { clauseNumber, } \\
\text { type, } \\
\text { domain, } \\
\text { name }\end{array}$ & control() \\
\hline AnyCorrectiveAction & $\mathrm{BO}$ & $\begin{array}{l}\text { duration, } \\
\text { name, } \\
\text { type, } \\
\text { serialNumber, purpose }\end{array}$ & $\begin{array}{l}\text { produceLog() } \\
\text { resultEvidence() }\end{array}$ \\
\hline AnyEvidence & BO & $\begin{array}{l}\text { timestamp, } \\
\text { assertion, } \\
\text { reliability }\end{array}$ & prove0 \\
\hline AnyLog & BO & $\begin{array}{l}\text { name, } \\
\text { size, } \\
\text { media, } \\
\text { logNumber, } \\
\text { timestamp }\end{array}$ & record 0 \\
\hline Loss & IO & $\begin{array}{l}\text { cost, } \\
\text { type }\end{array}$ & affectNegatively() \\
\hline Company & IO & $\begin{array}{l}\text { name } \\
\text { location } \\
\text { objective }\end{array}$ & seekMaintenance() \\
\hline Policy & IO & $\begin{array}{l}\text { text, } \\
\text { clause, } \\
\text { name }\end{array}$ & institureWarning() \\
\hline Counseling & IO & $\begin{array}{l}\text { duration, } \\
\text { reason, } \\
\text { domain }\end{array}$ & $\begin{array}{l}\text { motivateEmployee() } \\
\text { improveMentality() }\end{array}$ \\
\hline Attendance & IO & $\begin{array}{l}\text { registerName, } \\
\text { size }\end{array}$ & $\begin{array}{l}\text { showPresense() } \\
\text { keepTrack() }\end{array}$ \\
\hline BalanceSheet & IO & \begin{tabular}{|l} 
name, \\
day, \\
month, \\
year
\end{tabular} & $\begin{array}{l}\text { elicitateFinance() } \\
\text { storeRecord() }\end{array}$ \\
\hline Employee & IO & id, & undergoCounselling() \\
\hline
\end{tabular}




\begin{tabular}{|c|c|c|c|}
\hline & & name & \\
\hline Warning & IO & $\begin{array}{l}\text { type, } \\
\text { instruction }\end{array}$ & $\begin{array}{l}\text { inform() } \\
\text { issueWarning() }\end{array}$ \\
\hline \multicolumn{4}{|c|}{ Description } \\
\hline \multicolumn{4}{|c|}{$\begin{array}{l}\text { 1. AnyParty(Company) seeks Maintenance } \\
1.1 \text { which has AnyReason(Loss) } \\
1.2 \text { AnyReason(Loss) determines AnyType } \\
\text { TC: Maintenance is done by what? Who seeks Maintenance? Maintenance is based on what? } \\
\text { Why is Maintenance required? What determines AnyType? How? }\end{array}$} \\
\hline \multicolumn{4}{|c|}{$\begin{array}{l}\text { 2. Maintenance } \\
\text { 2.1 is achieved through AnyMechanism(Warning, AnyCorrectiveAction), } \\
2.2 \text { A Company seeks Maintenance, because of the problem of Loss and } \\
\text { 2.3 uses Warning to intimate to the Employee about it as it is being created, because of } \\
\text { Employee's low Attendance } \\
\text { TC: } \text { what achieves Maintenance? How is Maintenance achieved? Why is Maintenance needed? }\end{array}$} \\
\hline \multicolumn{4}{|c|}{$\begin{array}{l}\text { 3. AnyType } \\
3.1 \text { specifies AnyEntity which } \\
3.2 \text { is used by AnyMechanism(Warning, AnyCorrectiveAction) } \\
3.3 \text { to achieve Maintenance }\end{array}$} \\
\hline \multicolumn{4}{|c|}{$\begin{array}{l}\text { 4. AnyParty(Company) } \\
\text { 4.1 defines AnyCriteria(Policy) } \\
\text { 4.2 Company defines a Policy which } \\
\text { 4.3 Governs the code of conduct of Employees } \\
\text { 4.4 And describes the process of issuing Warning to Employees } \\
\text { TC: who defines AnyCriteria? AnyParty does what? Based on? }\end{array}$} \\
\hline $\begin{array}{r}\text { An } \\
5 \\
5 \\
5\end{array}$ & $\begin{array}{l}\text { ia do } \\
\text { ria? }\end{array}$ & at controls A & $\begin{array}{l}\text { on) } \\
\text { ow Attendance, since it is creating } \\
\text { ? Is this control needed? How? Who }\end{array}$ \\
\hline 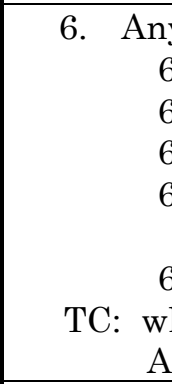 & $\begin{array}{l}\text { Cou } \\
\text { yEvi } \\
\text { o An } \\
\text { ande } \\
\text { get } p \\
\text { g int } \\
\text { e is i } \\
\text { recti } \\
\text { nyLo }\end{array}$ & $\begin{array}{l}\text { (Attendance) } \\
\text { BalanceShee } \\
\text { Counseling } \mathrm{v} \\
\text { ctive and un } \\
\text { ction } \\
\text { sed as a resu } \\
\text { on result? } \\
\text { hat do they d }\end{array}$ & $\begin{array}{l}\text { ected in balance sheet) } \\
\text { expectations of the Company along } \\
\text { es it give rise to? What produces }\end{array}$ \\
\hline $\begin{array}{r}\text { An } \\
7 \\
7 \\
\text { TC: } \mathrm{w} \\
\mathrm{M}\end{array}$ & $\begin{array}{l}\text { Mech } \\
\text { Mai } \\
\text { Any }\end{array}$ & $\begin{array}{l}\text { nce } \\
\text { lanism? Hov }\end{array}$ & related to what? What achieves \\
\hline
\end{tabular}


8. AnyLog(BalanceSheet)

8.1 helps prove that any AnyCorrectiveAction(Counselling) was taken

8.2 undergoing Counseling helps the Employee to

8.3 improve Attendance

8.4 which reduces Loss

8.5 which is shown by the BalanceSheet

TC: what gives a proof of AnyCorrectiveAction? What proves that AnyCorrectiveAction was taken? What else does this do? How?

\section{Alternatives}

1. Company seeks Maintenance to the problem of Loss

2. which is being generated due to lack of Attendance

3. Company institutes a policy for incentives for better attendance

4. and raises the salaries

5. which motivates the Employee to have a better attendance record

6. which helps in reducing the Loss to the Company.

\subsubsection{Behavior Diagram}

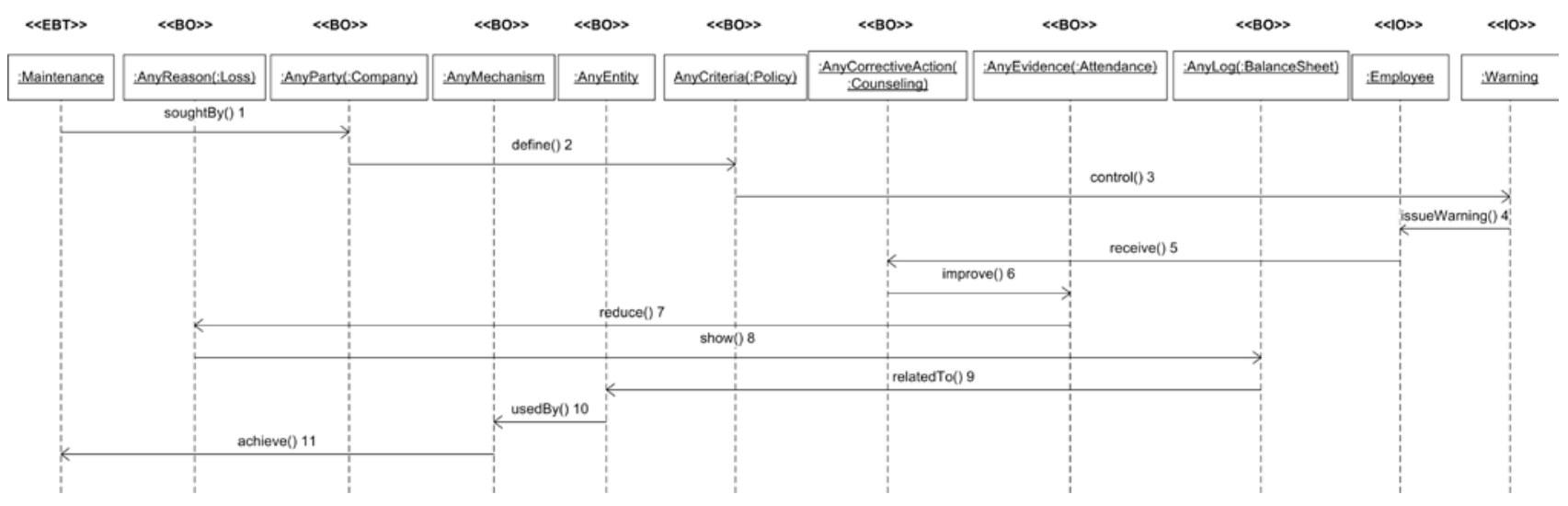

Figure 3 - Application 1 Sequence Diagram

\subsection{Modeling Issues, Criteria, and Constraints}

\subsubsection{Abstraction}

To design this project, we have used the software stability model, which consists of three parts. The Enduring Business Theme and the Business Objects form the essential parts of the model. They remain stable throughout any design and for any application. The IOs change for different applications. The EBT is the Enduring Business Concept, it is the Stable Analysis model and using this ensures that the ultimate goal of the system or business as such is never compromised, and that the system is modeled using that goal in mind. The EBT is then realized by using BOs or Business Objects, which are the workhorses for this EBT and these BOs provide hooks various IOs or Industrial objects to be attached to the pattern model according to the system that we are planning to design.

Here, the EBT is Maintenance, which serves as the goal for any such system and there are various BOs associated to it like AnyCorrectiveAction, AnyPolicy, AnyMechanism, AnyEvidence, AnyLog etc. they realize the enduring concept of Maintenance and according to the application that we want, e.g. reducing losses to an organization in this case, we attach the necessary IOs like Employee, Company, Loss, Policy etc. to these BOs, thereby modeling the entire system that is wanted.

\subsubsection{Modeling Heuristics}

While modeling the system, we need to follow certain modeling heuristics that enable a better system design. Few of them are as follows: 
No Star: There should not be any macho classes in the system, since having a star in the system calls for a focus of control on one class and it gets overloaded making the system prone to failures because of it. In our system, classes such as AnyCorrectiveAction and AnyCriteria have three connections already in the pattern class diagram, so we need to make sure that while connecting the IOs, we do not overload these classes by having more than one new connection to it, as that would result in an unbalanced situation and a macho class or a star.

No Dangling: No dangling classes should be left in the system. Dangling refers to a situation, where there is just one connection to a class. This situation is not good, since it creates a possibility of loss of control, as one the execution reaches that class it cannot be brought back. In this system, we have AnyEvidence dangling in the pattern class diagram, thus we need to make sure that we have at least one IO that is connected to this BO, so that AnyEvidence is not left in a dangling state.

No Sequence: There should not be any sequence because it results in many situations where the system may go into infinite loops without resulting in any tangible output. The figure below shows such a situation.

Moreover, if there is a sequence, then it may also mean that the selection of components and classes was not proper. Thus, when there is a sequence of say, 8 classes, it may rather be clubbed into one whole class or 2 classes that are communicating with each other, as shown in Figure 5.

General Design: The system should not be designed, while keeping in mind just one domain or application. We are looking at designing a system, which can be reused readily and can be applied to any domain in a domain independent fashion. Thinking about the possibilities in just a particular scenario will limit the capabilities of the pattern and will defeat the purpose of Stability Modeling.

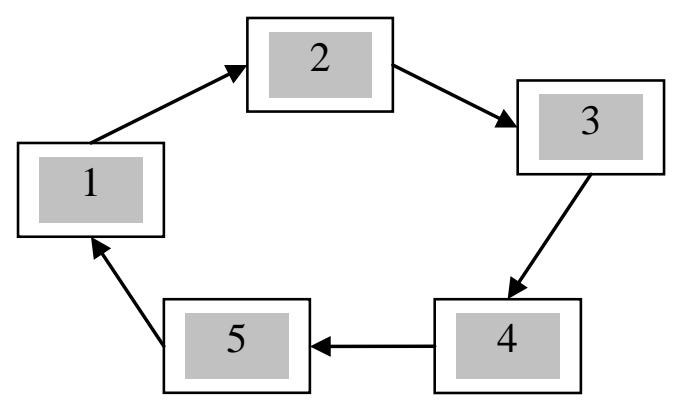

Figure 4 - Sequence (MUST be avoided)

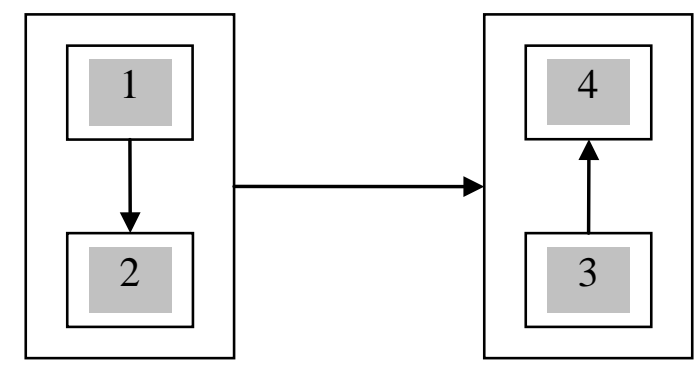

Figure 5 - Alternate view of an existing sequence

\subsection{Design \& Implementation Issues}




\subsubsection{Design Issues}

Selecting EBT \& BOs: To accurately model the Stable Design Pattern of AnyCorrectiveAction, the first and the most essential part is to choose the correct EBT for it. There are instances and goals which can be confused with the ultimate goal of a corrective action, like instead of choosing Maintenance, one may get confused with Betterment or Rectification, but AnyCorrectiveAction is not used for those situations, which require betterment as such. Maintenance is the correct EBT for the system. Also, the related BOs are very important as well. The BO AnyCorrectiveAction cannot realize the goal of Maintenance, without the presence of the correct and other relevant business objects in the system. AnyCorrectiveAction must be approved by AnyParty and must have AnyReason for it. The party is supposed to approve the corrective action. Then, AnyCorrectiveAction must produce AnyLog and AnyEvidence to support and show that the corrective action was taken and accomplished, so that the issuing authority gets to know that it was done. Hence, the correct selection of the EBT and other BOs in the system is critical for the correct behavior of the pattern.

\subsubsection{Implementation Issues}

Using Command Pattern: While implementing, instead of using approaches like inheritance etc., which make the system too brittle owing to the property of the methods of super class being transferred to the sub class even if some of them are needed or not, we can use the Command pattern to implement the system and this can be used while linking BOs to IOs.

In the Command pattern, there is a Command Interface and a ConcreteCommand implements this interface. There is a receiver that contains the specific method's code and is called by the ConcreteCommand upon request from the invoker, who wants to invoke that specific method. This allows us the flexibility of not having the clients tie to specific implementation code and they can just use the command to call a function without having to bother about its location of implementation details. On the service provider's end, it provides the system the flexibility to be maintained in a way that just the specific parts of the system are changed without affecting other classes in the system or the clients.

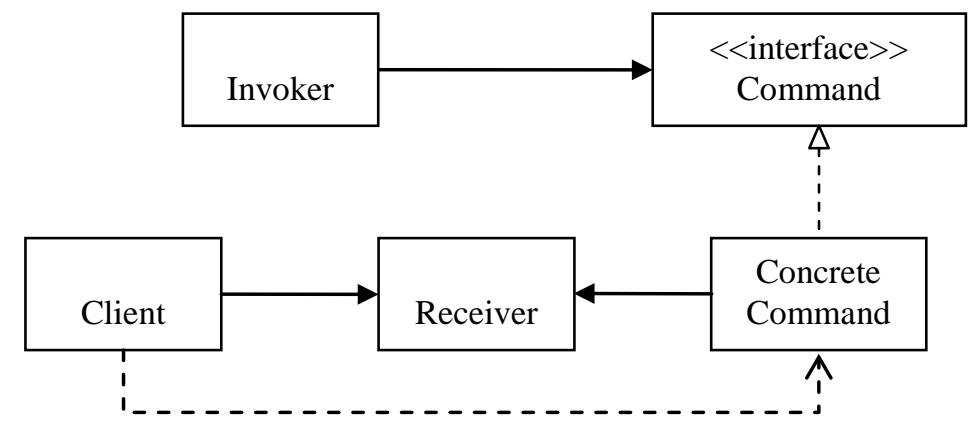

Figure 6 - The Command Pattern

In our pattern the Command pattern can be applied as follows:

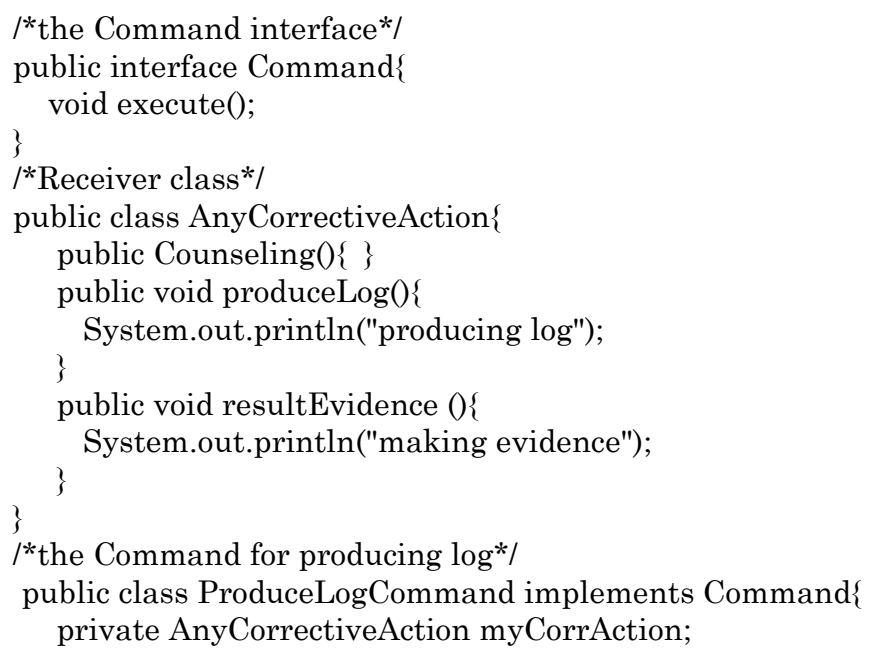




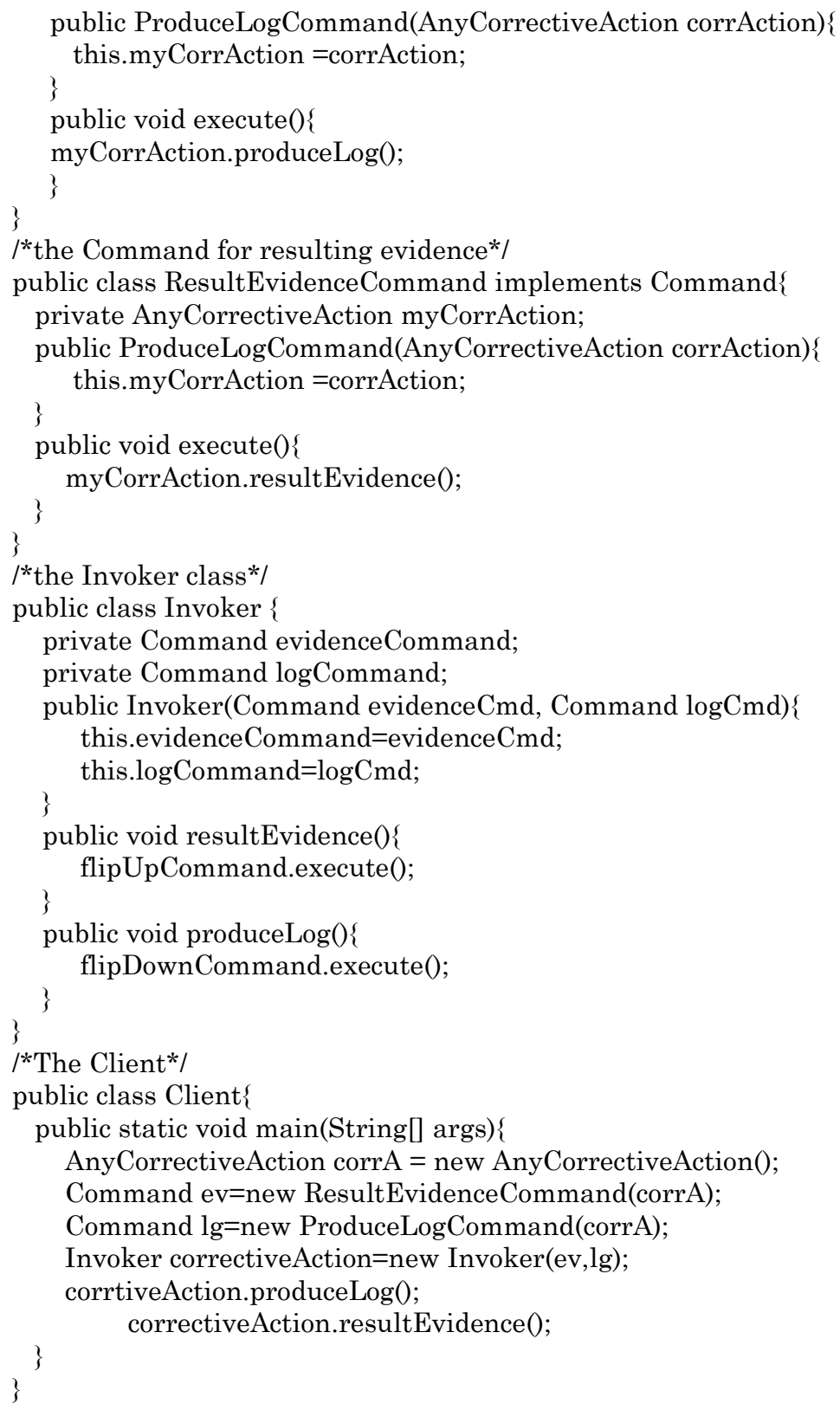

\subsection{Business Issues}

\section{Business Rules}

Business rules are constraints that govern the way in which a business is carried out. Our system pertains to the ultimate goal of Maintenance, which is achieved through AnyCorrectiveAction and other related BOs. Here are some business rules that apply to this system:

- AnyParty can define one or more AnyCriteria. Only specific kind of parties can create AnyCriteria. They are government agencies for administering the country for its well being. Scientists who discover and make theories or laws based on empirical vales and experiments, companies who make them for the employees to abide by for code of business and ethics.

- AnyMechanism is controlled by AnyCriteria. No mechanism being employed for corrective can go beyond the boundaries set forth by one or more governing criteria. 
- There must be AnyEvidence and AnyLog, which results from AnyCorrectiveAction, which is being taken that would serve as a proof to the authority that issued and approved it, of the fact that corrective action was taken.

- AnyParty is the governing authority that observes the situation and approves that AnyCorrectiveAction be taken. AnyCorrectiveAction cannot be taken without the permission and approval of the governing authority.

\subsection{Known Usage}

1. Corrective Action is a term which is more familiar in military. Many of the Military applications have been seen to use a system that would be comprised of this pattern, e.g. in a situation that involves the military to reroute or reframe its strategy of attack. This has to be authorized by the commanding authority and the corrective action of realigning the military to organize a coordinated and effective plan of action, will be taken.

1. The pattern has also found its implementation in the business world. "A corrective action is a change implemented to address a weakness identified in a management system. Normally corrective actions are implemented in response to a customer complaint, abnormal levels of internal nonconformity, nonconformities identified during an internal audit or adverse or unstable trends in product and process monitoring such as would be identified by SPC.”(from Wikipedia)

2. Any Company can use this pattern to correct any behavior that leads to some sort of non-conformity to its rules and policies. As shown in the application example in this paper, the non-pattern oriented approach to come up with a system that does something like what this pattern does, can be seen very frequently in the business world.

3. Another known usage of the pattern is among educational institutions like universities to take corrective actions in order to counter the problems like plagiarism or discrimination, misconduct etc. For example, Stanford University has a complete guideline for conducting Corrective Action for addressing conduct and performance issues. Here, a set criteria, policies and purpose statements are provided that determine why as well as when such an action has to be taken, within what limits and under which set of policies. At the end a report of the actions taken has to be provided with formal documentation and notice to all the parties involved. [8]

4. The pattern can really be applied to any situation or domain, where there is a need for Maintenance, due to some reasons of non conformance to the desired behavior. Though traditionally used in military applications, it can be exported over to other domains as well.

2.14. Tips and Heuristics

- Betterment, as good as it may sound to be an EBT for AnyCorrectiveAction pattern, actually it isn't. Maintenance is a better choice.

- To fit the EBT of Maintenance and to realize the pattern AnyCorrectiveAction, the other BOs in the system must be appropriate and in context with AnyCorrectiveAction or else the true purpose of this may not be solved.

- There were certain BOs chosen earlier, but discarded later, because they did not fit in the pattern or were redundant; also, it was hard to find IOs for those BOs.

- While developing the IO, when the connections are not possible, it means that something is missing in the BO or the chosen EBT is not correct. This situation was faced, when a particular BO was never used in any application or an application could not be linked.

\section{CONCLUSION}

Stability modeling approach towards modeling the system is a big leap ahead, from the Traditional way of modeling the system, because it provides us a pattern that can fit more than one, in fact many applications, in a domain independent fashion. While in traditional modeling, we developed a system with its specific purpose in mind and modeled just one application of a pattern, it was not possible to model to any other scenario. But, the stability model keeps in mind the ultimate goal, and it models a system 
accordingly, thus making it a flexible and a more maintainable system. Here, we saw that corrective action is a technique applied mostly in military and academia. The pattern has been seen in these domains earlier but has not been documented yet in a formal way. When we model it by using the stability modeling technique, we can take it and apply it to domains like business as shown in the applicability part. It was an eye opener to realize the flaws that existed in the current approaches and to experience a better way of software modeling. This work presents a stable core design for any system that would be built for doing corrective action.

Future work can include the development of the many different IOs that would apply the presented pattern in many different application contexts across various domains picture of where it fits in along with other patterns, which together solve the issue at hand. Moreover, the paper also presents a structure of the pattern and how it fits in with other patterns to solve the problem at hand and this can be a first step towards realizing a complete pattern language.

\section{REFERENCES}

[1] "Corrective Action", n.d. [Online]. Available: Onelook.com http://onelook.com/?w=corrective +action\&ls=a [Accessed May 26, 2010].

[2] M. E. Fayad, and A. Altman, "Thinking objectively: an introduction to software stability", Communications of the ACM, vol. 44, no. 9, pp. 95, Sep. 2001.

[3] H. Hamza, A. Mahdy, M. E. Fayad and M. Cline, "Extracting Domain-Specific and Domain-Neutral Patterns Using Software Stability Concepts", Lecture Notes in Computer Science, vol. 2817. Berlin / Heidelberg: Springer, 2003, pp. 191-201.

[4] "Correction", n.d. [Online]. Available: MSN Encarta, http://encarta.msn.com/encnet/features/dictionary/DictionaryResults.aspx? refid=1861600494 [Accessed May 26, 2010].

[5] "Correction", n.d. [Online]. Available: YourDictionary .com: http://www.yourdictionary.com/correction [Accessed May 26, 2010].

[6] M.E. Fayad, H.A. Sánchez and H.S. Hamza. A Pattern Language for CRC Cards. Proceedings of Pattern Language of Programs' 2004 (PLOP’04), Monticello- Illinois, USA, Sept. 2004.

[7] M.E. Fayad, Haitham Hamza, and Huáscar A. Sánchez "A Pattern for an Effective Class Responsibility Collaborator (CRC) Cards". Proceedings of the 2003 IEEE International Conference on Information Reuse and Integration (IRI'03), Las Vegas, Nevada, pp. 584-587, October 2003.

[8] "Addressing Conduct \& Performance Issues", Administrative Guide Memo 22.15, Stanford University, September 1, 2010 [Online], Available: http://adminguide. stanford.edu/22_15.pdf [Accessed Oct 15, 2010]. 